\title{
Territorialidade da rede de atenção psicossocial de um município do interior da Bahia
}

RESUMO I O presente estudo teve como objetivo identificar qual a territorialidade da Rede de Atenção Psicossocial numa cidade do interior da Bahia no período de 2012 a 2015, na perspectiva de Território em Rede. Para tanto, desenvolveu-se uma pesquisa de abordagem qualitativa apoiada no levantamento bibliográfico de textos e utilizou-se com estratégia metodológica a pesquisa de campo através de entrevistas direcionadas aos usuários e/ou familiares, profissionais e gestores. Identificou-se os dispositivos de atendimento necessários para a integração da rede psicossocial, resultando em sua Territorialidade, que é o nexo que estabelece com a Rede de Atenção Psicossocial do Território. A avaliação da RAPS permitiu conhecer a realidade construída através das experiências e interação de pessoas e tecnologias de informação.

Palavras-chaves: Territorialidade; Saúde Mental; Saúde Pública.

ABSTRACT | The objective of this study was to evaluate the Psychosocial Care Network in a municipality in the interior of Bahia from the period of 2012 to 2015 from the perspective of Network Territory. A bibliographical survey and a field research were conducted through interviews directed to users and / or family members, professionals and managers. The necessary care devices for the integration of the psychosocial network were identified, resulting in its Territoriality, which is the nexus that establishes with the Network of Psychosocial Care of the Territory. The evaluation of the RAPS allowed to know the reality built through the experiences and interaction of people and information technologies.

Keywords: Territoriality; Mental health; Públic Health.

RESUMEN | Este estudio tuvo como objetivo identificar la territorialidad de la Red de Atención Psicosocial en un municipio del interior de Bahía en el período de 2012 a 2015 en la perspectiva de Territorio en Red. Se realizó un levantamiento bibliográfico e investigación de campo a través de entrevistas dirigidas a usuarios y / o familiares, profesionales y gestores. Se identificaron los dispositivos de atención necesarios para la integración de la red psicosocial resultando en su Territorialidad, que es el nexo que establece con la Red de Atención Psicosocial del Territorio. La evaluación de la RAPS permitió conocer la realidad construida a través de las experiencias e interacción de personas y tecnologías de información.

Descriptores: Territorialidad; Salud Mental; Salud Pública.

\section{Adriana Antonia de Oliveira}

Mestra em Planejamento Territorial e Desenvolvimento Social - Universidade Católica do Salvador. Especialista em Urgência e Emergência - FSSS. Especialista em Saúde Mental - FSSS. Especialista em Obstetrícia - CBPEX. Professora da Faculdade Santo Antonio de Alagoinhas - BA. Professora da Faculdade Dom Luiz de Orleans e Bragança. Enfermeira do município de Entre Rios - BA e Cipó - BA.

Recebido em: 31/05/2019

Aprovado em: 15/01/2020

INTRODUÇÃO

m dezembro de 2011, foi san-
cionada a Lei federal n. 3.088
que instituiu a Rede de Atenção Psicossocial, visando promover a integração entre os pontos de atenção das redes de saúde no território composta pelos componentes de atenção básica, atenção psicossocial especializada, atenção de urgências e emergências, atenção residencial de caráter transitório e atenção hospitalar ${ }^{(1)}$.

A rede de atenção psicossocial passa a designar um novo paradigma de cuidados em saúde mental, promovendo um aperfeiçoamento técnico e institucional do tratamento e tendo efeitos positivos também na cidadania brasileira ${ }^{(2)}$.

Dentre as diversas compreensões teóricas sobre território, assumiu-se nesta pesquisa, o território enquanto espaço em que se constrói e não apenas um con- trole físico, mas também, laços de identidade territorial que, explicado de modo composto com a noção de redes, possibilita compreender-se as articulações de territorialidades, bem como, suas estruturações internas ${ }^{(3)}$.

Mais recentemente, nas sociedades ditas "de controle" ou "pós-modernas" vigora o controle da mobilidade, dos fluxos (redes) e, consequentemente, das conexões - o território passa então, gradativamente, de um território mais "zonal" ou de controle de áreas para um "território-rede" ou de controle de redes. Aí, o movimento ou a mobilidade passa a ser um elemento fundamental na construção do território ${ }^{(4)}$.

Desse modo, a construção e o controle de conexões e rede (fluxos) de pessoas e informações, bem como, a identificação de grupos tornam-se elementos fundamentais para a construção do território em rede. Não se trata apenas de teorizar 
sobre as inovações em saúde, mas de investigar a inovação enquanto ação concreta que tem no respeito ao indivíduo a relevância da inovação, o que requer mais do que logística operacional em rede, e que a abordagem territorial oferece elementos para avaliar tendo em vista o conteúdo identitário.

Assim é que, o objetivo identificar qual a territorialidade da Rede de Atenção Psicossocial numa cidade do interior da Bahia no período de 2012 a 2015, na perspectiva de Território em Rede. Ao situar-se teórica e institucionalmente como tema de pesquisa, a inquietação pelos desafios de conhecer a cobertura da Rede de Atenção Psicossocial (RAPS), questionou-se a partir da perspectiva de território em rede, qual a territorialidade com que se constitui a Rede de Atenção Psicossocial em um município do interior da Bahia?

\section{MÉTODO}

\section{Tipo de Estudo}

Trata-se de uma pesquisa realizada através de procedimentos qualitativos e quantitativos, mas para construção textual desse artigo, optou-se por um recorte que contemplou apenas os qualitativos. Assim sendo, o método de abordagem foi o de análise e síntese.

\section{Instrumentos utilizados}

Avaliando as ações dos gestores municipais, assim como, dos profissionais atuantes nos pontos de atenção identificados, além da percepção dos usuários, buscou-se informações através de entrevistas, com questionários semiestruturados, considerando um roteiro de questões norteadoras sobre a Rede de Atenção Psicossocial. O local de pesquisa foram os pontos de atenção à saúde, o que facilitou a percepção da realidade vivida, além de evitar vieses que afastassem o propósito principal da pesquisa, direcionados aos usuários e/ ou seus familiares, profissionais e gestores, Identificados como: "Profissional", profissionais de Enfermagem neste artigo foram identificados "Enfermagem", "Gestor" "Usuário/Familiar" e "Usuário". Campo de estudo desta pesquisa é a Rede de Atenção Psicossocial do município no interior da Bahia, dentre os serviços de saúde, apenas os pontos de atenção à saúde previstos na portaria $\mathrm{n}^{\circ} 3.088 / 2011$ e identificados no site do Cadastro Nacional de Estabelecimentos de Saúde (CNES). Foram identificados os pontos de atenção à saúde através de siglas: (ABU) Atenção Básica localizada na Zona Urbana e (ABR) Atenção Básica localizada na Zona Rural; (SAMU) Serviço de Atendimento Móvel de Urgência; (AMB) Ambulatório de Psiquiatria; e (CAPS) Centro de Atenção Psicossocial, e numerados conforme ordem de pesquisa, como por exemplo: "Enfermagem 01 ABR".

O Hospital do município não foi incluído na pesquisa de campo, porque se encontrava em processo de troca de gestão, modificando uma parcela considerável de funcionários, o que dificultou, assim, o acesso para as autorizações, bem como, o relato de experiência nos anos de recorte da pesquisa.

\section{Amostra}

No total foram 25 entrevistados, de modo a garantir a qualidade da pesquisa qualitativa, sendo 08 profissionais de saúde, 15 usuários ou familiares e 02 coordenadores da Rede de Atenção Psicossocial.

Adotou-se a oferta dos serviços, como critério de inclusão de informantes institucionais, e a demanda pelo serviço, como critério de inclusão de informantes sujeitos individuais e familiais. Como decorrência desse critério, foram excluídos quaisquer informantes que não estivessem vinculados como ofertantes ou demandantes do sistema, sendo assim escoIhidos e identificados.

Os aspectos legais de pesquisa em saúde, regulamentados pela Resolução n466, de 12 de dezembro do ano 2012, aprovada pelo Conselho Nacio- 
nal de Saúde, foram rigorosamente observados. Submetido ao Comitê de Ética da Universidade Católica de Salvador, obteve parecer aprovado por meio da Plataforma Brasil, com o número do parecer: 1.390.40.

Os participantes da pesquisa foram esclarecidos sobre os procedimentos adotados durante toda a pesquisa e sobre os possíveis riscos e benefícios. Foram garantidos, sob a ótica do indivíduo e das coletividades, referenciais da bioética tais como a autonomia, não maleficência, beneficência, justiça e equidade, o sigilo de todas as informações referidas, bem como o direito de recusar-se a participar da pesquisa a qualquer momento, sem que isso traga prejuízo ao entrevistado; com isso foram assegurados os direitos e deveres dos participantes da pesquisa.

\section{Período de coleta de dados}

O período escolhido para a análise abrange os anos entre 2012 e 2015, período que sob a norma oficial instituiu a Rede de Atenção Psicossocial em 23 de dezembro de 2011. O período abordado permitiu uma avaliação pioneira da atuação dos profissionais, levantando as principais questões que impedem, dificultam ou facilitam o exercício dos trabalhos de atendimento às pessoas em sofrimento psíquico, configurando sua dinâmica, para a qual a interdisciplinaridade na abordagem territorial poderá subsidiar uma atuação profissional mais efetiva.

\section{Análise e tratamento dos dados}

Todas as entrevistas foram gravadas, transcritas e analisadas. Foi utilizada a análise de conteúdo. "As diferentes fases da análise de conteúdo, organizam-se em torno de três polos cronológicos: 1) a pré análise; 2) a exploração do material; 3) o tratamento dos resultados, a inferência e a interpretação" (6).

O método de tem por finalidade para a descrição objetiva do conteúdo das comunicações, cumprindo-se as desenvolvendo-se em três fases estabelecidas, a saber: Pré-análise, compreendendo a fase de organização, sendo realizada a leitura de todo o material; para a execução da segunda fase que é a exploração do material envolvendo recorte, enumeração e classificação das categorias conforme conteúdo das perguntas e das respostas ${ }^{(5)}$.

Estabeleceu-se na dissertação ${ }^{(6)} 4$ categorias e 10 subcategorias para a Territorialidade em Rede, e para este artigo, devido a necessidade de síntese, foram abordadas em 3 categorias que mostra a relação estabelecida com a RAPS dos gestores, profissionais, usuários e/ou familiares.

E a terceira e última fase foi o tratamento dos dados. Para o tratamento dos dados foram comparadas as respostas dos diferentes sujeitos da pesquisa, a partir de das categorias territoriais e interpretada donde interpretou-se para a descrição da rede no território construído e descreveu-se através das categorias de territorialidade, a como relação dos entrevistados com a rede em suas indagações e sugestões de aprimoramento da RAPS.

\section{RESULTADOS}

Gondim $^{(7)}$ descreveu a territorialidade em saúde como a prática, a reorganização do processo de trabalho em saúde, que no caso da saúde mental poderá ser associado à visão de $\operatorname{Costa}^{(8)}$ quando afirma que, para entender a política de saúde mental, deve-se reaproximar do âmbito local, enfatizando o território e atentando para as suas múltiplas concepções.

Interações sociais mediadas pelo espaço $^{(9)}$, âmbito local em múltiplas concepções de território(10) e condicionantes territoriais nas formas de trabalhar em equipe de maneira integral dão conteúdo à reorganização do processo de trabalho em saúde que Gondim ${ }^{(7)}$ descreve como territorialidade e que nesta pesquisa é assumida como categoria para avaliar a dinâmica da RAPS.

A territorialidade foi examinada na rede psicossocial através dos dispositivos de atendimento necessários para a integração da rede, pela percepção dos gestores, profissionais e usuários do serviço, ou seja, a relação estabelecida nos pontos de atenção à saúde ligada ao território em rede.

\section{DISCUSSÃO}

Categoria 1: Relação estabelecida com a RAPS - Gestores

Quando se trata da escuta da demanda usuária do serviço de saúde mental, o gestor 02 relata que foi iniciada no segundo semestre de 2015 um diálogo com os presidentes das associações de bairro e outras instituições que atendem às demandas de saúde mental para ampliar as parcerias. O gestor 01 refere que há uma busca pela escuta individualizada dos usuários nas próprias unidades, e informações para o acesso a ouvidoria do município para acolher as necessidades dos usuários para o aprimoramento da rede:

[...] nós sempre estamos ouvindo as queixas dos usuários, para a cada dia estarmos tentando melhorar nosso atendimento [...] temos também a ouvidoria do município, que a gente tem cartaz espalhado na unidade, para se o usuário não quiser se expor no momento, estar procurando a ouvidoria, para ter as explicações, e a coordenação de todas as unidades, está sempre ciente que qualquer paciente que tiver queixa, eles vão estar recebendo, para a gente trabalhar em cima da queixa daquele usuário. (Gestor 01)

Segundo a gestão iniciada no segundo semestre de 2015, um diálogo com os presidentes das associações de bairro e outras instituições que atendem às demandas de saúde mental para ampliar as parcerias foi aberto, fortalecendo e ampliando os dispositivos da Rede Psicossocial. Além disso, ambos os gestores entrevistados passaram as mesmas informações sobre as medidas implantadas para a melhoria, demonstrando 
boa articulação no planejamento da rede psicossocial.

Categoria 2: Relação estabelecida com a RAPS Profissionais

No que concerne a cooperação dos profissionais na RAPS, conforme entrevistas, está diretamente relacionada à ligação estabelecida entre os profissionais e o território em rede psicossocial. Os mais participativos e mais conscientes mostraram disposição e interesse para a integração da rede, realizando atendimentos, encaminhado e acompanhando periodicamente os usuários.

Sim, sim a gente faz todos, a médica periodicamente, ela sempre atende, e é assim, tem alguma necessidade que eu vejo que não é demanda médica que é mais alguma orientação, questão da família também eu costumo atender, costumo realizar visitas, o quando tem algum paciente que teve algum problema especial, os agente comunitários sinalizam, a gente visita, conversa com a família, outro serviço que a gente tem aqui é o CRAS, que a gente conseguiu fazer essa interlocução com as meninas e ai a assistente social, peço pra elas fazerem as visitas e elas fazem. (Enfermagem ABR 12).

Todavia, contrastando com a dinâmica da "Enfermagem ABZR 12", outros mostraram "falta de afinidade" como desafio para a participação no território em rede psicossocial estudado. Foi levantado por eles mesmos a dificuldade já citada na contra referência estar ligada à "falta de interesse" de alguns profissionais:

[...] eu tive outra dificuldade, é até porque, vamos dizer, não é minha praia, o público que eu tenho de HIPERDIA é que vem só pra ver alimentação saudável e a parte da prescrição médica [...] nunca também me interessei porque não é uma coisa, como eu te falei, não me atrai, eu posso atender sim, mas não é um público que me atrai, tipo assim, eu vou me aprofundar em saúde mental, eu acho bonito quem gosta, pra mim saúde da mulher é o máximo, entendeu? E cada um sabe o que realmente, não é menosprezando ninguém, eu acho lindo, mas pra mim cada um sabe o que gosta, o que tem afinidade. (Enfermagem $A B \cup$ 05)

Acho que...Dificuldade é a contra referência, eu acho que um pouco de desinteresse [...]. (Enfermagem ABR 11)

Diante disso, os profissionais indicaram conforme sua vivência cotidiana com a rede, as principais dificuldades de integração da rede, como também, sugeriram e confirmaram quais medidas meIhorariam a fluidez da rede psicossocial. As reuniões não foram citadas apenas com o NASF, mas também demonstraram interesse para o aprimoramento de conhecimento através dos especialistas em saúde mental:

Gostaria que tivesse mais acesso aos especialistas, e também fazer um trabalho em grupos, até mesmo que os especialistas passassem alguma coisa pra gente, isso eu acho que seria ótimo, sabe por que? A gente trabaIharia melhor, saberia um pouco mais, o que fazer, e diminuiria um pouco a demanda lá no CAPS. Então eu acho que seria ótimo que fizessem isso com a gente. (Enfermagem ABU 06).

Nesse processo de capacitação foi citado pelo profissional CAPS 08, também outros pontos de atenção para o CAPS, capacitando os profissionais do CAPS, na detecção precoce de outras patologias clínicas e encaminhamento adequado, para "costurar a rede" em todos os sentidos, citando como exemplo, uma experiência:

Tivemos um enfermeiro aqui pra discutir essa questão da Hanseníase [...] Então pra gente não ficar aqui tão fechadinho no nosso casulo de que só é saúde mental, porque é uma crítica que eu faço também, às vezes a gente acha que a unidade de saúde da família não se preocupa com saúde mental, as vezes acha que a gente se preocupa apenas com isso, não apenas no sentido que é pouco, mas que a gente também às vezes não atenta pra essas questões [...]. (Profissional CAPS 08)

Os profissionais do CAPS demonstram iniciativa e busca constante de superação das dificuldades encontradas:

"[...] a gente tenta atravessar essas dificuldades usando a criatividade [...]. (Enfermagem CAPS 01).

Em suma, os profissionais demonstraram disposição e interesse para a integração da rede conforme identificação e capacitação ao serviço em rede de atenção psicossocial. Apesar de citadas as dificuldades organizacionais de acesso a exames e medicamentos solicitados, o transporte para realização de visitas, também foi enfatizada a importância da conscientização e sensibilização entre a comunidade, familiares e profissionais para chegar a soluções.

Categoria 3: Relação estabelecida com a RAPS - Usuários e/ou familiares

Os usuários se mostraram atentos aos limites e possibilidades do serviço de saúde mental do município. Discutiram questões importantes para a melhoria relacionada ao serviço de saúde mental, como a importância de haver mais médicos, aumentar o número de visitas domiciliares aos usuários que frequentam a unidade, sobre a importância de haver 
mais guardas na unidade para organização e controle da unidade, aumentar o número de oficinas, colchões para os acompanhantes dos usuários em acolhimento noturno, e melhorar a alimentação, exemplificado nas citações abaixo:

É ter mais visita, que por enquanto as visitas são poucas, mas tendo mais visita pode melhorar muito mais. (Usuário/Familiar 03).

Uma coisa importante que eu tenho a falar pra todo mundo aqui ouvir, o CAPS aqui, ele tem que melhorar não é só na medicação, no atendimento, mas também na alimentação ( Usuário/Familiar 01).

Eu acho que precisa mais de mais condições para pessoas, as pessoas que ficam acolhidos aqui, e os acompanhantes dos acolhidos podiam ter colchões pra ficar acompanhando os acoIhidos porque as pessoas que chegam ficam dormindo sentados nas cadeiras, esperando, as vezes ficam dias aqui 8 a 10 dias (Usuário/Familiar 02).

Na percepção dos usuários, em relação às consultas agendadas e a suficiência do serviço, mostraram-se conhecedo- res da rotina de marcação nas unidades, e sua periodicidade, se sentindo acolhidos no sistema de marcação:

É de três em três meses que a gente vem, e se tiver algum problema a gente pode vir antes que eles atendem a gente. (Usuário 02).

Enfim, os usuários demonstraram boa relação com os profissionais do CAPS, sentimento de acolhimento, e despertos ao funcionamento do serviço de saúde mental do município.

\section{CONCLUSÃO}

A RAPS necessita das medidas político - institucionais e recursos como para a efetivação da rede psicossocial, que ainda está em construção enfrentando os desafios do estigma da herança psicossocial, poderá ser fortalecido pelo poder público em sua capacidade de coordenação estratégica, de organicista de ações, sancionando decisões estruturantes através dos financiamentos adequados.

Além das condições de trabalho, a relação estabelecida com o território em rede está diretamente ligada ao compromisso dos gestores, profissionais da saúde mental, e outros profissionais interessados em prestar um bom serviço a rede, esta tem sido "tecida" diariamente, a cada experiência, e aos poucos a rede tem se fortalecido.

De forma positiva os profissionais em sua maioria se mostraram bem interagidos ou interessados no aprendizado, no entanto uma parcela ainda precisa passar por sensibilização, e todos e forma contínua de capacitação. Os gestores demonstraram comprometimento com o fortalecimento da rede, através da estratégias de escuta para a construção da RAPS. E os usuários se mostraram atentos ao funcionamento do serviço de saúde mental, indicando medidas de melhorias, relacionadas à estrutura, atendimento e acessibilidade.

Em síntese, a avaliação da Rede de Atenção Psicossocial do município estudado na perspectiva de Território e Territorialidade em Rede, permitiu conhecer a realidade construída através das experiências e interação de pessoas.

A caracterização da sua dinâmica de funcionamento em rede de atenção psicossocial com base no território, e dos seus dispositivos de integração foram passos fundamentais para conhecer a Rede em seus aspectos, e compreender que a extensão de sua cobertura e sua territorialidade são interdependentes para garantia da efetividade do direito à saúde para todos os seus usuários.

\section{Referências}

1. Brasil. Portaria $n^{\circ} 3.088$, de 23 de dezembro de 2011 . Institui a Rede de Atenção Psicossocial para pessoas com sofrimento ou transtorno mental e com necessidades decorrentes do uso de crack, álcool e outras drogas, no âmbito do Sistema Único de Saúde. Diário oficial da União. 2011[Acesso em 13 de junho 2015] Disponivel em: http://bvsms.saude.gov.br/bvs/saudelegis/gm/2011/prt3088_23_12_2011.html

2. Tenorio F. A reforma psiquiátrica brasileira, da década de 1980 aos dias atuais: história e conceitos. História, Ciências, Saúde-Manguinhos [revista em internet] 2002;10 (9): [Acesso em: 13 de junho de 2015].25-59. Disponível em: http://www.scielo.br/pdf/hcsm/v9n1/a03v9n1.pdf

3. Haesbaert R. Territórios alternativos. São Paulo: Contexto, 2002.

4. Haesbaert R. 0 mito da desterritorialização. Rio de Janeiro: Bertrand Brasil, 2004.

5. Bardin F. Análise de conteúdo [livro online]. Lisboa: Edições 70; 1977. [Acesso em: 7 nov. 2016]. Disponível em http://docslide.com.br/education/ bardin-laurence-analise-de-conteudo.html\#.
6. Oliveira AA. Territorialidade em rede de atenção psicossocial no Município de Alagoinhas - Ba. Salvador. Dissertação [Mestrado em Planejamento Territorial e Desenvolvimento Social, Planejamento Territorial e Desenvolvimento Social] - Universidade Católica do Salvador; 2016.

7. Gondim R et al. Qualificação de gestores do SUS. Rio de Janeiro: EAD/ ENSP, 2009, (1):402.

8. Costa LA, Brasil FD. Cidade, territorialidade e redes na política de saúde mental. Caderno de Terapia Ocupacional 2014; 22(2): 435-442.

9. Lages VN et al. Territórios em movimento: cultura e identidade como estratégia de inserção competitiva. Brasília: Relume Dumará; SEBRAE, 2004. [Acesso em: 30 out. 2015]. Disponível em: http://bis.sebrae.com.br/ GestorRepositorio/ARQUIVOS_CHRONUS/bds/bds.nsf/E1C3CE6A43DBDB3203256FD6004907B7/\%24File/NT00031436.pdf.

10. Alencar CMM. Metropolitan Rurality in the dynamic territorial local. Interações: Campo Grande 2010; 11(1): 23-32. 